\title{
Intracytoplasmic Morphologically selected Sperm Injection vs Intracytoplasmic Sperm Injection: A Retrospective Analysis
}

\author{
${ }^{1}$ Rutvij Jay Dalal, ${ }^{2}$ Hrishikesh D Pai, ${ }^{2}$ Nandita P Palshetkar, ${ }^{3}$ Akanksha P Mishra \\ ${ }^{1}$ Fellow of National Board in Reproductive Medicine, Lilavati Hospital and Research Center, Mumbai, Maharashtra, India \\ ${ }^{2}$ Senior Consultant, IVF, Lilavati Hospital and Research Center, Mumbai, Maharashtra, India \\ ${ }^{3}$ Senior Embryologist, IVF, Lilavati Hospital and Research Center, Mumbai, Maharashtra, India
}

Correspondence: Rutvij Jay Dalal, Fellow of National Board in Reproductive Medicine, Lilavati Hospital and Research Center Mumbai, Maharashtra, India, e-mail: rutvij.dalal@gmail.com

\section{ABSTRACT}

This study was conducted to determine the efficacy of using the technique of intracytoplasm morphologically selected sperm injection (IMSI) as compared to the conventionally used intracytoplasmic sperm injection ((ICSI) procedure. IMSI, as the name suggests, is the technique of selecting the most normal looking sperm by magnifying it to about 7200 times. A total of 192 patients who underwent the treatment of in vitro fertilization (IVF) with us by either one of the two procedures of IMSI or ICSI over a period of 18 months were included in the study. Out of these 92 were included in the IMSI group while 100 others in the ICSI group according to our inclusion and exclusion criteria. The pregnancy rate among patients who underwent IMSI was found to be significantly higher (52.1\%) as compared to those with ICSI (36\%). Furthermore, even the implantation rates were higher in the IMSI group (30.12\%) than the ICSI (19.93\%) group.

Keywords: High magnification, ICSI, IMSI, Male infertility, MSOME, Sperm selection, OAT syndrome.

\section{INTRODUCTION}

A new method of high magnification $(\times 7200)$ motile sperm organellar morphology examination (MSOME) published by Bartoov et al $^{1}$ in 2002 showed that the fertilization rate is directly proportional to the incidence of morphologically normal spermatozoa. This was found to be statistically significant.

The main purpose of our retrospective study was to determine the efficacy of the intracytoplasmic morphologically selected sperm injection (IMSI) procedure compared to the conventional intracytoplasmic sperm injection (ICSI) procedure in the treatment of patients with severe oligoasthenoteratozoospermia (OAT) and/or at least two previous failed IVF attempts.

\section{MATERIALS AND METHODS}

\section{Patient Selection}

From January 2009 up to August 2010, 192 couples were observed. Observation criteria included: (1) Patients with severe oligoasthenoteratozoospermia and/or at least two previous failed pregnancy outcomes. (2) An undetected female factor or no obvious pathology in the female partner. (3) Woman's age 35 years or younger. (4) Couples with more than or equal to

Date of Received: 05-04-11

Date of Acceptance: 06-04-11

Date of Publication: September 2011 two years of infertility. Exclusion criteria: (1) Patients with unexplained infertility with less than two previous in vitro fertilization (IVF) attempt failures. (2) Any detected pathology in the wife/female. (3) Patients with established azoospermia even with testicular biopsy. (4) Patients with two or more previous consecutive miscarriages.

Patients were divided into following groups as follows: group 1(IMSI) $(\mathrm{n}=92)$, group 2 (ICSI) $(\mathrm{n}=100)$. These two groups were further subdivided into subgroups as: (a) With no previous IVF attempt failure (b) with one previous IVF attempt failure and (c) with two or more IVF attempt failures. All the patients underwent the same mid-luteal phase down-regulation protocol with GnRH agonist ('long' protocol) and started stimulation with recombinant FSH from day 2 of cycle.

\section{Semen Evaluation and Preparation}

Semen analysis was done in the laboratory just prior to preparation for injection. The diagnosis of severe oligoasthenoteratozoospermia was confirmed by the World Health Organization (WHO) criteria for sperm concentration $\left(<5 \times 10^{6} / \mathrm{ml}\right)$ and motility ( $<20 \%$ progressive) (WHO, 1992), and strict Kruger's criteria $(<4 \%$ normal forms) for sperm morphology evaluation. ${ }^{2}$

We use only freshly ejaculated semen for sperm preparation for performing ICSI/IMSI. The preparation of the semen was performed using pellet-swim up technique for normal samples (WHO criteria) and pellet reconstitution technique for severe 
oligozoospermic samples. The washing of semen was done with MediCult HEPES buffered flushing medium MediCult (Catalog no ....) and the pellet was overlaid/reconstituted with embryo culture medium (Vitrolife catalog no...).

\section{IMSI Sperm Selection}

The glass Petri dish (Willco-dish; Willco wells BV, Amsterdam, The Netherlands) for IMSI was prepared as follows: (i) In the center, three observation droplets of polyvinylpyrrolidone medium (MediCult medium) were made (ii) surrounding the PVP drops were $7 \mu \mathrm{l}$ drops of flushing medium to host the oocytes that were to be injected with the selected sperms.

The droplets were overlaid with sterile liquid paraffin (MediCult).

The sperm cell suspension obtained after semen preparation was used for real-time high magnification $\mathrm{MSOME}^{3}$ that was performed on the observation droplets by means of a Narishige micromanipulator, with attached inverted microscope (Nikon Eclipse, TE2000S, Tokyo, Japan). The images were captured by a high definition USB2.0 camera 3 MPx and visualized on a monitor screen with diagonal dimension of $42 \mathrm{~cm}$.

Sperms with obvious defects like pin head, amorphous head, large mid-piece, double tail/head were not evaluated. In this study, it is assumed that the sperms exhibiting normal morphology according to MSOME criteria have normal nuclear DNA content and organization, which have been reported to exert a significant effect on ICSI fertilization rate and embryo development.

To perform the evaluation, the following steps were taken:

1. First, the seemingly normal sperms were immobilized.

2. Five to six of them were placed in a row one below the other in the microscopic field.

3. They were magnified real time $(\times 6000)$ and then an image was clicked.

4. With the help of a computer program, the measurement of the image of the sperm was taken.

5. The strict descriptive criteria for normally shaped nuclei were based on those defined by scanning electron microscopy, i.e. smooth, symmetric and oval configuration. ${ }^{1,3}$ Only motile spermatozoa with normal head dimensions (length 4.5 to $5.0 \mu \mathrm{m}$, width 3.0 to $3.5 \mu \mathrm{m}$ ) were selected (Fig. 1).

6. The sperms thus selected were further magnified 7200 times and observed for vacuoles. Those sperms with no or a maximum of one vacuole $(<1.0 \mu \mathrm{m})$ were selected for injected into the oocytes. The nuclear chromatin content was considered normal if it contained no more than one vacuole, which occupies $<4 \%$ of the nuclear area (Fig. 2).

Two embryologists worked together for analyzing the sperms to minimize the chances of human error.

\section{Microinjection}

The transferred, retrieved, cumulus-free ova were placed into drops of MediCult buffered medium prepared in the same glass dish with the recipient droplet. Sperm cells morphologically selected for IMSI were then finally used for injection into the oocytes by classical ICSI. ${ }^{4}$ This procedure was performed using Narishige Micromanipulation System using Injection Pipette (COOK'S MPIP catalog no....) and holding pipette (COOK'S HPIP catalog no.....) at $400 \times$ magnification.

\section{Embryo Culture}

The injected oocytes were immediately transferred to a center well dish (Falcon 3037), incubated in $0.8 \mathrm{ml}$ of IVF medium (Vitrolife) covered with $0.4 \mathrm{ml}$ of mineral oil (MediCult) at $37^{\circ} \mathrm{C}$ with an atmosphere of $5 \% \mathrm{CO}_{2}$. Three to four oocytes were cultured per dish.

Embryo transfer was done on day 2 or 3 after visual grading and selection of best embryos (when more than three embryos were formed). Usually three embryos per patient were transferred except in cases when only one or two embryos were formed, in which case whichever embryos obtained were transferred. The pregnancy rate was calculated per transfer attempt.

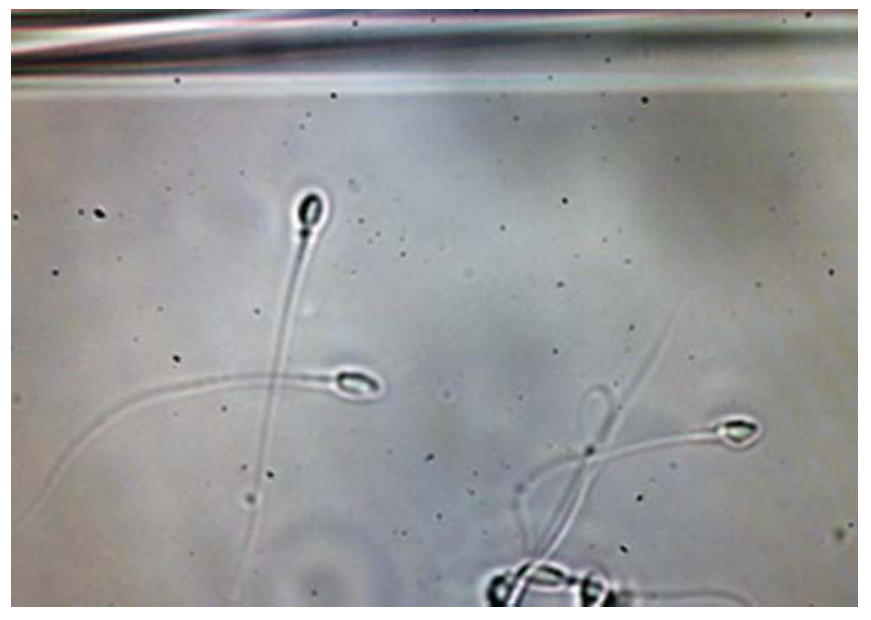

Fig. 1: Appearance of sperms with IMSI procedure (x 7200 magnification)

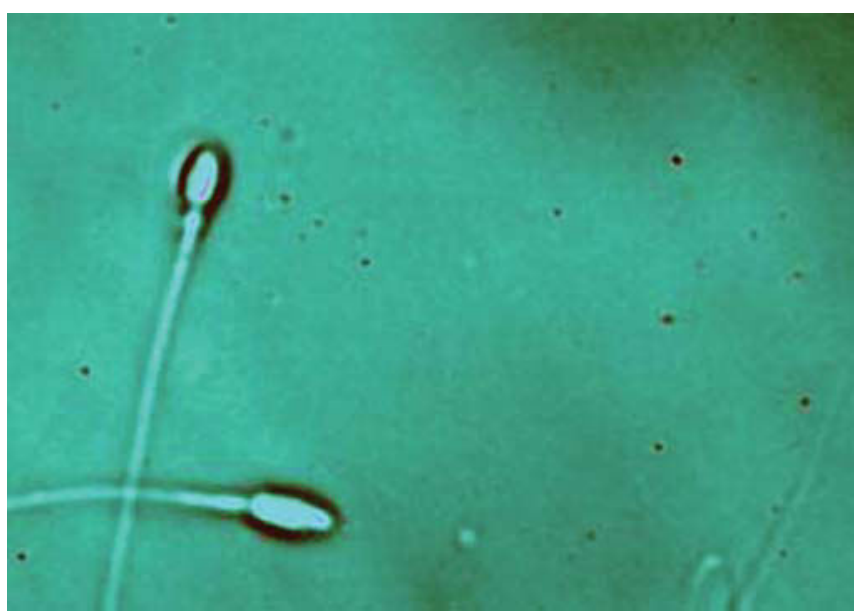

Fig. 2: Sperms magnified 7200 times to identify vacoules 


\section{STATISTICAL METHODS}

Data are presented as mean \pm SD. Statistical evaluation was performed with the Student's t-test to compare continuous variables while a Chi-square test was used to compare discrete variables. $\mathrm{p}<0.05$ was considered statistically significant.

\section{RESULT}

There were no statistical differences between the main groups in terms of mean age, number of previous failed ICSI attempts, number of recovered oocytes and transferred embryos (Table 1).

A total of 24 IMSI and 18 ICSI patients attained positive $\beta$-hCG followed by fetal heart beat detection by the sixth week. Up to now, the IMSI procedure has resulted in seven deliveries of a total of 11 healthy babies (four twins), 13 ongoing pregnancies and four miscarriages. As for the ICSI group, six healthy babies were born (2 twins), four miscarriages occurred and 10 pregnancies are still ongoing. All miscarriages took place during the first trimester.

By comparing groups 1 and 2, IMSI pregnancy and implantation rates appear to be significantly higher than those for ICSI (pregnancy rate 52.17 vs 36\%, and implantation rate $30.63 \%$ vs $19.83 \%)$.

$\mathrm{PN}=$ pronucleate. Continuous variables are presented as means \pm SD.

$$
{ }^{\mathrm{a}} \mathrm{p}<0.05 ;{ }^{\mathrm{b}} \mathrm{p}<0.05
$$

Upon comparison of the two techniques by subgroups with different previous failed attempts (Table 2), the following pregnancy rate results were obtained: (i) Subgroup 1C vs subgroup 2C: $25 \%$ vs $51.35 \%$, p $<0.05$; (ii) no statistical difference was observed between subgroup $1 \mathrm{~A}$ and subgroup $2 \mathrm{~A}(40 \%$ vs $50 \%)$, and between subgroup $1 \mathrm{~B}$ and subgroup $2 \mathrm{~B}$ (44.12\% vs 54.84\%) (Table 3), although the clinical outcome was clearly in favor of the IMSI method.

Table 1: Comparison of fertilization, pregnancy and implantation rates arising from intracytoplasmic sperm injection (ICSI) and intracytoplasmic morphologically selected sperm injection (IMSI) groups

\begin{tabular}{|c|c|c|c|c|c|}
\hline & \multirow{2}{*}{$\begin{array}{c}\text { Group } 1 \\
I C S I(n=100)\end{array}$} & \multirow{2}{*}{$\begin{array}{c}\text { Group } 2 \\
\text { IMSI }(n=92)\end{array}$} & \multicolumn{3}{|c|}{ Unpaired t-test applied } \\
\hline & & & $t$-value & $p$-value & Difference \\
\hline Mean age (years) & $32.91 \pm 3.30$ & $32.65 \pm 3.23$ & -0.530 & 0.5963 & NS \\
\hline Number of previous ICSI failures & $1.65 \pm 1.57$ & $1.69 \pm 1.69$ & 0.17 & 0.8652 & NS \\
\hline Number of oocytes recovered & $10.39 \pm 3.04$ & $9.58 \pm 3.34$ & -1.759 & 0.0802 & NS \\
\hline Number of injected oocytes & $8.92 \pm 0.26$ & $8.85 \pm 0.29$ & -1.763 & 0.0794 & NS \\
\hline Number of 2 PN zygotes & $6.66 \pm 0.42$ & $7.23 \pm 0.45$ & 9.078 & $<0.0001$ & NS \\
\hline Number of transferred embryos/patient & $2.81 \pm 0.67$ & $2.82 \pm 0.68$ & 0.103 & 0.9184 & NS \\
\hline Clinical pregnancy rate (\%) & $36 / 100(36)^{a}$ & $48 / 92(52.17)^{\mathrm{a}}$ & $4.457^{c}$ & 0.0347 & $\mathrm{~s}$ \\
\hline Implantation rate $(\%)$ & $56 / 281(19.93)^{b}$ & $78 / 259(30.12)^{\mathrm{b}}$ & $6.960^{\mathrm{C}}$ & 0.0083 & $S$ \\
\hline & & 2 twins & 4 twins & & \\
\hline
\end{tabular}

aPregnancy detected by observing a viable pregnancy (with heart beat) on ultrasound examination

bImplantation rate calculated per embryo transferred

${ }^{\mathrm{C}}$ Chi-square test applied with continuity correction; NS: Nonsignificance; S: Significance

Table 2: Comparison of pregnancy arising from intracytoplasmic sperm injection (ICSI) and intracytoplasmic morphologically selected sperm injection (IMSI) subgroups with a different number of previous IVF failures

\begin{tabular}{clrr}
\hline Subgroup & \multicolumn{1}{c}{ Rate } & Group 1, IMSI & Group 2, IMSI \\
\hline Subgroup A (0 ICSI failures) & Pregnancy & 12 & 12 \\
& No pregnancy & 18 & 12 \\
& Total & 30 & 24 \\
Subgroup B (1 ICSI failure) & Percentage of pregnancy rate & $40 \%$ & $50 \%$ \\
& Pregnancy & 15 & 17 \\
& No pregnancy & 34 & 31 \\
Subgroup C (2 ICSI failures) & Total & $44.12 \%$ & $54.84 \%$ \\
& Percentage of pregnancy rate & 9 & 19 \\
& Pregnancy & 27 & 37 \\
& No pregnancy & 36 & $51.35 \%$ \\
\hline
\end{tabular}

Table 3: Statistical analysis of the above-mentioned subgroups in terms of pregnancy rate

\begin{tabular}{lcccc}
\hline Chi-square tests & Value & $d f$ & $p$-value & Association \\
\hline Subgroup A (0 ICSI failures) & 0.211 & 1 & $0.646^{\mathrm{a}}$ & $\mathrm{NS}$ \\
Subgroup B (1 ICSI failure) & 0.378 & 1 & $0.538^{\mathrm{a}}$ & $\mathrm{NS}$ \\
Subgroup C (2 ICSI failures) & 4.302 & 1 & $0.038^{\mathrm{a}}$ & $\mathrm{S}$ \\
\hline
\end{tabular}

${ }^{\mathrm{a}}$ Chi-square test applied with continuity correction; NS: Nonsignificance; S: Significance 


\section{DISCUSSION}

Evolutionary biologists have developed several hypotheses that link sperm selection to the inheritance of superior fitness traits (i.e. disease resistance, offspring survival and fecundity). It is believed that the healthiest sperm is naturally selected by the ovum for fertilization. Hence, since the advent of IVF, semen parameters have played an important role in determining the outcome of treatment. ${ }^{5-9}$ However, with the introduction of microinsemination techniques ${ }^{4,10-12}$ sperm morphology evaluation has lost its importance as a routine diagnostic criteria in infertility management. Sperm selection is based on the judgment of an embryologist who selects the most normallooking spermatozoon available under $\times 200 / 400$ magnification. Though some researchers feel that the outcome of ICSI is not related to basic sperm parameters. ${ }^{13-16}$ Numerous studies have established that the correct selection of a morphologically normal sperm greatly enhances the outcome of ICSI. ${ }^{17-19}$

However, the dependence on the expertize of the embryologist for the selection of a morphologically normal sperm is very subjective and not completely devoid of errors. To overcome this hurdle a number of advanced microscopes have been recently developed to magnify sperms thousands of times to clearly observe the morphology.

Benjamin Bartoov in Israel was the first to use the technique of IMSI in 2003. He selected patients with at least two previous ICSI failures. He used the MSOME criteria to select morphologically normal sperms and injected them into the eggs. They suggest that ICSI pregnancy rates may be affected by subtle morphological malformations of the sperm nucleus, which embryologists may not detect during routine ICSI sperm selection. The study compared 50 couples in each group. They found that the couples who underwent IMSI had a significantly higher pregnancy rate $(66 \%)$ than those who underwent conventional ICSI (33\%). However, this was based on a small sample size. ${ }^{3}$

Since then numerous studies have been performed with encouraging results in favor of the IMSI procedure. ${ }^{20-22}$

The concept of IMSI can be better appreciated if we understand the fact that after all in cases of natural conception, the most healthy and morphologically and physiologically normal sperm is selected out of the millions to attain fertilization of a single egg. This can be extrapolated into saying that if we want to achieve successful in vitro fertilization, it will do us good to be able to select the sperm which is the most normal in all aspects. However, there is no denying the complexity of natural sperm selection mechanisms which is supported by the fact that despite the normally large numbers of spermatozoa in an ejaculate, only a minority are able to meet the stringent requirements needed to fertilize an egg. This leads to the obvious conclusion that sperm quality assessment methods in the laboratory would improve if they could incorporate aspects of these selective processes. However, one has to consider that there are a number of physiological, morphological and genetic aspects that come in the natural selection of sperms that the right mix still remains elusive despite years of research.
The best method of selection of the best sperm can be via the process of elimination. In case when a sperm is being singled out for injection into an egg, selection can be done based on the assumption that the sperms that pass the MSOME test are closer to normal than the ones that do not. ${ }^{1}$ The morphology of the sperm organelles defined by this method was found to have the highest predictive ability for male fertility potential. ${ }^{3}$

Our results demonstrate that the morphological state of the entire sperm cell, and especially of its nucleus, has predictive value for fertilization, pregnancy outcome, or both following ICSI. We applied the MSOME criteria prior to ICSI for 92 couples (group 1) as compared to the 100 couples (group 2) and found spermatozoa free of any specific morphological malformations exhibited the highest positive correlation with ICSI pregnancy rate. These present results are not in accordance with some authors who believe that ICSI outcome is not related to strict morphology of the spermatozoon used for microinjection. ${ }^{23-26}$

In reviewing the literature, there are conflicting studies. For example, few studies indicate that fertilization, embryo development and pregnancy is possible even if normal spermatozoa are not available (100\% of teratozoospermia). ${ }^{13,27-29}$ Whereas a few other studies have found that fertilization, pregnancy and also implantation rates are adversely affected when sperms with severe abnormalities were used for ICSI. ${ }^{17,19,30}$ One more study in 2001 also concludes into saying that sperm morphology is significantly correlated to the percentage of embryos developing to the blastocyst stage $(30.3 \%$ vs $51.9 \%)$ and to high quality blastocysts $(13.6 \%$ vs $28.2 \%){ }^{18}$

\section{CONCLUSION}

This study is a unique retrospective study that proves the effectiveness of IMSI over ICSI to benefit patients who have severe oligoteratozoospermia and/or repeated IVF/ICSI failures. The patients with more than one failed attempt at IVF/ICSI seem to have increased their chances of pregnancy two-fold after undergoing IMSI. The implantation rate per embryo also significantly increased from 19.93 to $30.12 \%$. However, one should bear in mind the small sample size. The authors opine that a controlled prospective study involving a large sample size is in order to be able to analyze the true statistical significance of the benefit of IMSI over ICSI.

\section{REFERENCES}

1. Bartoov B, Berkovitz A, Eltes F, Kogosowski A, Menezo Y, Barak Y. Real-time fine morphology of motile human sperm cells is associated with IVF-ICSI outcome. Journal of Andrology 2002;23:1-8.

2. Kruger TF, Acosta AA, Simmons KF, Swanson JR, Matta JF, Oehninger S. Predictive value of sperm morphology in in vitro fertilization. Fertility and Sterility 1988;49:112-17.

3. Bartoov B, Berkovitz A, Eltes F, Kogosovsky A, Yagoda A, Lederman $\mathrm{H}$, et al. Pregnancy rates are higher with intracytoplasmic morphologically selected sperm injection than with conventional intracytoplasmic injection. Fertility and Sterility 2003;80:1413-19. 
4. Palermo G, Joris H, Devroey P, Steirteghem V AC. Pregnancies after intracytoplasmic injection of single spermatozoon into an oocyte. Lancet 1992;340:17.

5. Kruger TF, Menkweld R, Stander FSH, Lombard CJ. Sperm morphological features as a prognostic factor in IVF. Fertility and Sterility 1986;46:1118-23.

6. Kruger TF, Acosta AA, Simmons KF, Swanson RJ, Matta JF, Veeck LL, et al. New method of evaluating sperm morphology with predictive value for human in vitro fertilization. Urology 1987;3:248-51.

7. Parinaud J, Mieusset R, Vieitez G, Labal B, Richoilley G. Influence of sperm parameters on embryo quality. Fertility and Sterility 1993;60:888-92.

8. Eilish TD, Lewis SEM, McNally JA, William T. In vitro fertilization and pregnancy rates: The influence of sperm motility and morphology on IVF outcome. Fertility and Sterility 1998;70: 305-14.

9. Ombelet W, Bosmans E, Janssen M, Cox A, Vlasselaer J, Gyselaers W, et al. Semen parameters in a fertile versus subfertile population: A need for change in the interpretation of semen testing. Human Reproduction 1997;12:987-93.

10. Ng SC, Bongso A, Sathananthan H, Chan CL, Wong PC, Hagglund L, et al. Pregnancy after transfer of sperm under zona. Lancet 1988;2:790.

11. Fishel S, Antinori S, Jackson P, Johnson J, Rinaldi L. Twin birth after subzonal insemination. Lancet 1990;335: 722-23.

12. Antinori S, Panci C, Caffa B, Selman HA, DanI G, Pavova A de, et al. The RAPRUI. Center experience: From SUZI, through lasers to ICSI using spermatozoa with broken tails. Human Reproduction 1995;10:489-91.

13. Nagy ZP, Liu J, Joris H, Verheyen G, Tournaye H, Camus. The result of intracytoplasmic sperm injection is not related to any of the three basic sperm parameters. Human Reproduction 1995;10:1123-29.

14. Peter Svalander1, Ann-Helene Jakobsson, Ann-Sofie Forsberg, Anna-Carin Bengtsson. The outcome of intracytoplasmic sperm injection is unrelated to 'strict criteria' sperm morphology Human Reproduction 1996;11(5):1019-22.

15. Mansour RT, Aboulghar MA, Serour GI, Amin YM, Ramzi AM. The effect of sperm parameters on the outcome of intracytoplasmic sperm injection. Fertil Steril 1995;64: 982-86.

16. Oehninger S, Chaturvedi S, Toner J, Morshedi M, Mayer J, Lanzendorf S, et al. Semen quality: Is there a paternal effect on pregnancy outcome in in vitro fertilization/intracytoplasmic sperm injection? Hum Reprod 1998;13:2161-64.

17. Kahraman S, Akarsu C, Cengiz G, Dirikan K, Sozen E, Can B, et al. Fertility of ejaculated and testicular megalohead spermatozoa with intracytoplasmic sperm injection. Human Reproduction 1999;14:726-30.
18. Miller JE, Smith TT. The effect of intracytoplasmic sperm injection and semen parameters on blastocyst development in vitro. Human Reproduction 2001;16:918-24.

19. De Vos A, Van De Velde H, Joris H, Verheyen G, Devroey P, Van Steirteghem A. Influence of individual sperm morphology on fertilization, embryo morphology, and pregnancy outcome of intracytoplasmic sperm injection. Fertility and Sterility 2003;79:42-48.

20. Berkovitz A, Eltes F, Ellenbogen E, Peer S, Feldberg D, Bartoov B. Does the presence of nuclear vacuoles in human sperm selected for ICSI affect pregnancy outcome? Human Reproduction 2006a;21:1787-90.

21. Berkovitz A, Eltes F, Lederman H, Peer S, Ellenbogen A, Feldberg B, et al. How to improve IVF-ICSI outcome by sperm selection. Reproductive BioMedicine Online 2006b;12:634-38.

22. Hazout A, Dumont-Hassan M, Junca AM, Cohen BP, Tesarik J. High-magnification ICSI overcomes paternal effect resistant to conventional ICSI. Reproductive BioMedicine Online 2006;12:19-25.

23. Oehninger S, Veeck L, Lanzendorf S, Maloney M, Toner J, Mausher S. Intracytoplasmic sperm injection: Achievement of high pregnancy rates in couples with severe male factor infertility is dependent primarily upon female not male factors. Fertility and Sterility 1995;64:977-81.

24. Kupker W, Schulze W, Diedrich K. Ultrastructure of gametes and intracytoplasmic sperm injection: The significance of sperm morphology. Human Reproduction 1998;13 (Suppl 1):99-106.

25. Host E, Ernst E, Lindenberg S, Smidt-Jensen S. Morphology of spermatozoa used in IVF and ICSI from oligozoospermic men. Reproductive BioMedicine Online 2001;3:212-15.

26. Celik-Ozenci C, Jakab A, Kovacs T, Catalanotti J, Demir R, Bray-Ward P, et al. Sperm selection for ICSI: Shape properties do not predict the absence or presence of numerical chromosomal aberrations. Human Reproduction 2004;19:2052-59.

27. Tasdemir I, Tasdemir M, Tavukcuoglu S, Kahraman S, Biberoglu K. Effect of abnormal sperm head morphology on the outcome of intracytoplasmic sperm injection in humans. Human Reproduction 1997;12:1214-17.

28. McKenzie LJ, Kovanci E, Amato P, Cisneros P, Lamb D, Carson SA. Pregnancy outcome of in vitro fertilization/ intracytoplasmic sperm injection with profound teratospermia. Fertility and Sterility 2004;82:847-49.

29. Mercan R, Lanzendorf SE, Mayer J Jr, Nassar A, Mausher SJ, Oehninger S. The outcome of clinical pregnancies following intracytoplasmic sperm injection is not affected by semen quality. Andrologia 1998;30:91-95.

30. Osawa Y, Sueoka K, Iwata S, Shinohara M, Kobayashi N, Kuji N, et al. Assessment of the dominant abnormal form is useful for predicting the outcome of intracytoplasmic sperm injection in case of severe teratozoospermia. J Assist Reprod genet. 1999;16:436-42. 